\title{
From mach cone to reappeared jet: What do we learn from PHENIX results on non-identified jet correlation?
}

\author{
Jiangyong Jia for the PHENIX Collaboration \\ Columbia University, New York, NY 10027 and Nevis Laboratories, Irvington, NY 10533, USA
}

\begin{abstract}
High $p_{T}$ jets are known to be strongly modified by the dense, strongly interacting medium created in heavy-ion collisions. The jet signal, extracted from two particle $\Delta \phi$ correlation, shows a systematic evolution of these modifications as function of $p_{T}$ and centrality. At intermediate $p_{T}$, both near side and away side correlations are modified. But the modifications are much stronger at the away side, resulting in a characteristic cone type of structure in central $\mathrm{Au}+\mathrm{Au}$ collisions. The robustness of cone structure is strengthened by studying the jet shape as function of angle relative to the reaction plane. As one increase the $p_{T}$ for BOTH hadrons, the cone structure seems to be filled up, and a peak structure appears on the away side. However, the interpretation of these results require careful separation of medium effect and surface bias.
\end{abstract}

PACS: $27.75 .-\mathrm{q}$

\section{INTRODUCTION}

High $p_{T}$ back-to-back jets are valuable probes for the sQGP [1] created in heavy-ion collisions at RHIC. Existing two particle jet correlation results from statistically limited RUN2 Au + Au data set revealed a strong interaction of the jets with the medium. On the one hand, jet correlation at high $p_{T}$ indicates a seemly complete disappearance of the away side jet signal [2]. On the other hand, jet correlation at low $p_{T}$ shows an enhancement of the away side jet yield [3] but a broadened jet shape [4]. Qualitatively, this is consistent with the energy loss picture, where the high $p_{T}$ jets are quenched by the medium and their lost energy enhanced the jet multiplicity at low $p_{T}$.

Equipped with excellent statistics from RUN4 $\mathrm{Au}+\mathrm{Au}$ and RUN5 $\mathrm{Cu}+\mathrm{Cu}$ data sets, we would like to gain further understanding on the interaction of the jets with the medium. We hope to address important questions such as: How the jet looses it's energy? How the lost energy get redistributed? How the medium responds to the jet? What happens to the higher $p_{T}$ jets? We attempt to address these questions using the non-identified charged hadron - charged hadron correlation results from RUN4 Au $+\mathrm{Au}$ data set.

\section{JET PROPERTIES AT INTERMEDIATE $P_{T}$}

Our analysis is based on 1 billion minimum bias events from $\mathrm{Au}+\mathrm{Au}$ collisions at $\sqrt{s}=$ $200 \mathrm{GeV}$. The correlation function $C(\Delta \phi)(\mathrm{CF})$ is defined as the ratio of same event pair distribution, $d N^{\text {pairs }} / d \Delta \phi$ to the mixed event pair distribution, $d N^{\text {mix }} / d \Delta \phi . d N^{\text {mix }} / d \Delta \phi$ 
reflects the level of combinatoric background and the geometrical acceptance [5]. In heavy-ion collisions, the CF can be expressed as the sum of jets and elliptic flow,

$$
C(\Delta \phi)=J(\Delta \phi)+\xi\left(1+2 v_{2}^{t} v_{2}^{a} \cos 2 \Delta \phi\right)
$$

The superscript $t$ and $a$ stand for the trigger and associated particles, $\xi$ is a normalization factor.
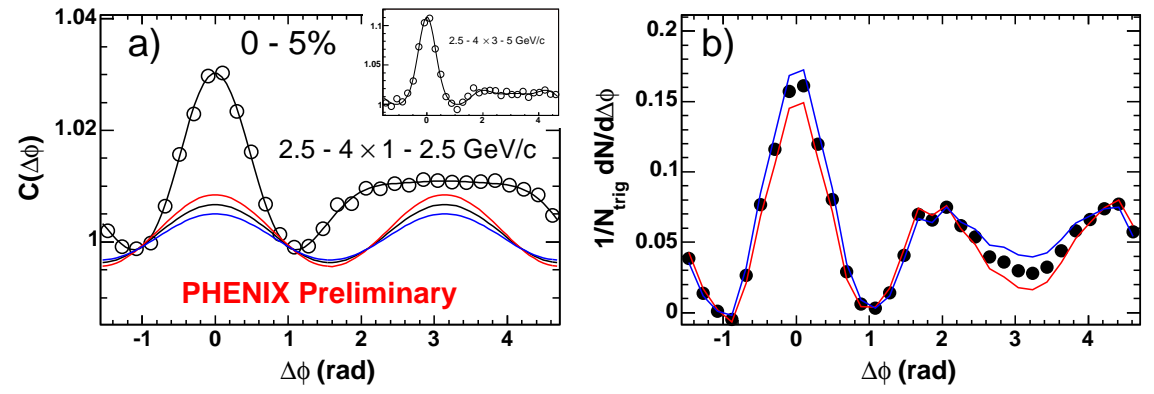

FIGURE 1. a) Correlation function in $0-5 \%$ centrality bin, the lines indicated the level of flow background and it's systematic error band, the insert is $\mathrm{CF}$ for higher $p_{T, \text { assoc }} . \mathrm{b}$ ) Correspondingly background subtracted per-trigger yield.

Fig. 1a shows the typical correlation function from central $\mathrm{Au}+\mathrm{Au}$ collisions. The away side shape is very broad and non-gauss like. It has a plateau that expands to about 2 radians and a possible small dip at $\pi$. The subtraction of the flow contribution (shown by the curves) only makes the dip deeper (Fig1b). $\xi$ is fixed by scaling the flow term to match the $\mathrm{CF}$, i.e. assuming $J=0$ at some $\Delta \phi$ (ZYAM assumption) [6]. The ZYAM procedure leads to a slight over-subtraction of jet yield, however since $2 v_{2}^{t} v_{2}^{a} \approx$ few $\%$, the over-subtraction mainly results in a vertical shift and does not affect the away side jet shape. The systematic error on $J(\Delta \phi)$ is dominated by the uncertainties on $v_{2}$.

PHENIX performed a systematic study of the jet shape and yield at intermediate $p_{T}$, as shown in Fig.2. There is a continues evolution of the split and the dip as function of centrality. The away side split is characterized by the split parameter $D$ [7], which is obtained by a double gauss fit on the away side. $D$ seems to turn on rather quickly as a function of centrality, and fall on a uniform curve as function of $\mathrm{N}_{\text {part }}$ for different collision energies and collision systems.
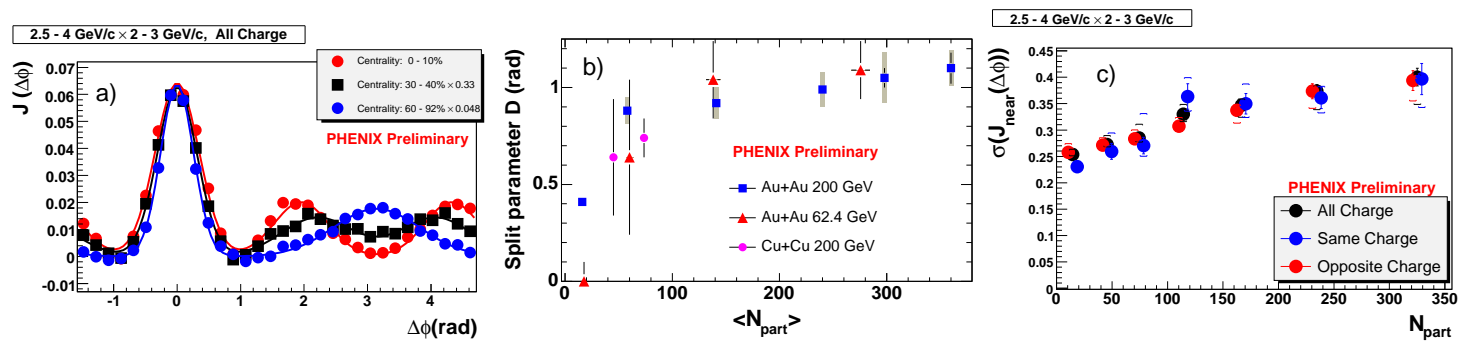

FIGURE 2. a) The correlation function for central, mid-central and peripheral bin in $\mathrm{Au}+\mathrm{Au}$. b) The away side "D" parameter as function of $N_{\text {part }}$ for several collision systems and energies. c) the near side width as function of centrality for same charged pairs, opposite charged pairs and all pairs in $\mathrm{Au}+\mathrm{Au}$. 


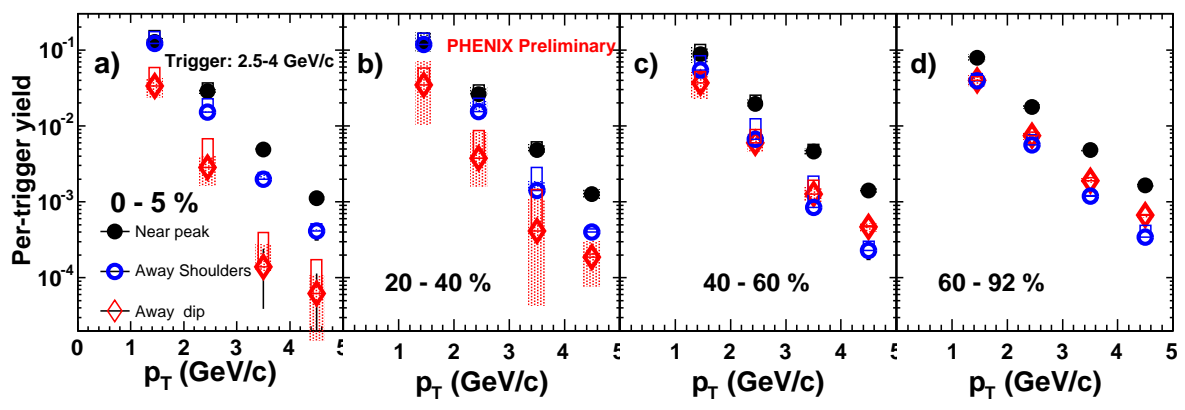

FIGURE 3. The yield for trigger $2.5-4 \mathrm{GeV} / c$ plotted as function of associated hadron $p_{T}$ for four different centrality bins.

What is the nature of the away side dip? Energy loss models that implement the jet broadening in a random walk manner can not describe the dip or the flat jet shape [8]. Models with Cherenkov gluons [9, 10] or medium dragging effect from flow [11] predict a cone or a broadening of the away side jet, but the predicted modifications depend strongly on momentum and are expected to disappear at large $p_{T}$. Casalderrey et. al. [12] proposed a 'mach cone'/'shock wave' mechanism to explain the away side jet shape. In this model, energetic jets, which travel faster than speed of sound $\left(c_{s}\right)$ in the medium, excite shock waves at an angle $\theta=\cos ^{-1}\left(c_{S} / c\right)$. The direction of the cone is independent of the $p_{T}$, but the width of the cone is predicted to narrow for higher $p_{T}$.

Fig. 2 also indicates a sizable broadening of the near side jet shape in central collisions. This modification is not as dramatic as that for the away side jet, most likely due to the surface emission bias [14] in which the average distance travelled by the near side jet is much smaller than that for the away side jet. However, the relatively small amount of medium that the near side jet has to go through could already lead to some broadening. On the other hand, the baryon yield is enhanced at intermediate $p_{T}$ in central collisions [15]. Since the near side jet structure could be different between baryon trigger and meson trigger [16], the broadening of the near side jet width could be a consequence of the strongly modified particle composition in central collisions.

To quantify the modifications of the jet shape, we study the jet yield in three different $\Delta \phi$ regions: near side jet region $(|\Delta \phi|<\pi / 3)$, the away side dip region $(|\Delta \phi-\pi|<\pi / 6)$, and the away side shoulder region $(|\Delta \phi-\pi \pm \pi / 3|<\pi / 6)$. The shoulder region is sensitive to the novel medium effects, while the dip region is sensitive to the punch through jet contribution. Fig. 3 plots the jet yields in the three regions as function of $p_{T}$ for four centralities. In $0-5 \%$ centrality bin, there is a large separation between the yields for the dip region and near side jet region, persistent to large $p_{T}$. In more peripheral collisions, the yield of the dip region becomes closer or even exceeds that for the shoulder region, consistent with the returning of the away side jet to a normal gauss shape. 

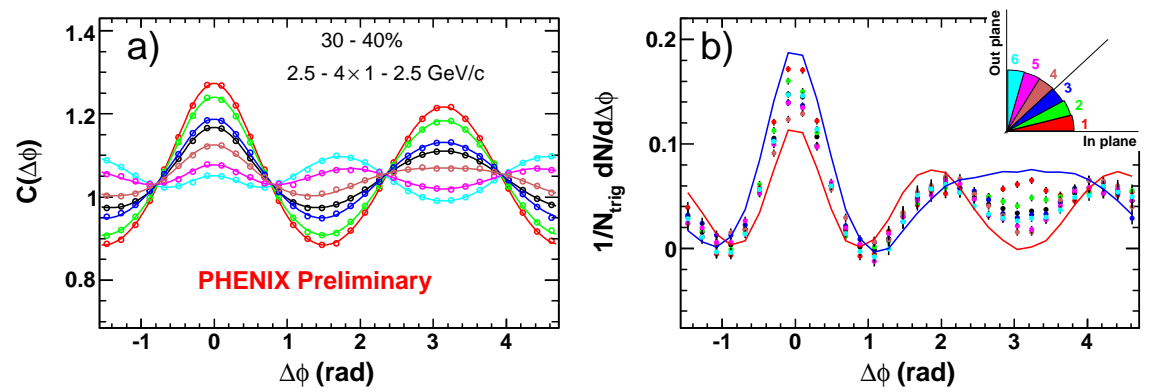

FIGURE 4. a) Correlation function for 6 trigger direction bins and the trigger integrated bin (the center curve). b) The flow subtracted per-trigger yields, the insert figure shows the 6 trigger bins.

\section{DEPENDENCE ON THE REACTION PLANE.}

The study of the jet yield as function of angle w.r.p to reaction plane is very important in the sense that it adds another dimension in controlling the path length dependence. It also provide additional constrains on the subtraction of the elliptic flow background. When the trigger particles are selected in a window centered around $\phi_{s}$ with a width of $\pm c$ with respect to the reaction plane, the pair distribution up to second order harmonics (without jet contribution) is [13]:

$$
\begin{gathered}
\frac{d N^{\text {pairs }}}{d \Delta \phi}=\frac{2 c}{\pi} B\left(a+2 v_{2}^{a} b \cos 2 \Delta \phi\right) \\
\left\{\begin{array}{l}
a=1+2 v_{2}{ }^{t} \cos 2 \phi_{s} \frac{\sin 2 c}{2 c}\langle\cos 2 \Psi\rangle \\
b=v_{2}{ }^{t}+\cos 2 \phi_{s} \frac{\sin 2 c}{2 c}\langle\cos 2 \Psi\rangle+v_{2}{ }^{t} \cos 4 \phi_{s} \frac{\sin 4 c}{4 c}\langle\cos 4 \Psi\rangle
\end{array}\right.
\end{gathered}
$$

$a$ is the combinatoric background level, and it is proportional to the number of trigger particles in the window. The correlation function without jet contribution is:

$$
C(\Delta \phi)=\frac{d N^{\text {pairs }} / d \Delta \phi}{d N^{\text {mix }} / d \Delta \phi}=\xi\left(1+2 v_{2}^{a} b / a \cos 2 \Delta \phi\right)=\xi\left(1+2 v_{2}^{a} v_{2, e f f}^{t} \cos 2 \Delta \phi\right)
$$

Where $v_{2, e f f}^{t}=b / a$ is the effective $v_{2}$ of the trigger particle in the window, and $\xi$ is the same normalization factor as in Eq.1. $\xi$ does not depend on the trigger direction.

We divide the trigger range, $[0, \pi / 2]$, into 6 bins. Each bin has a different flow background, which can be calculated from Eq.2. The measured correlation functions for 30$40 \%$ centrality bin are shown Fig. 4 . Several interesting features can be readily identified. The effective $v_{2, \text { eff }}^{t}$ changes dramatically from in plane to out of plane direction, but the six CFs cross each other at $\pm \pi / 4$ and $\pi \pm \pi / 4$, where the harmonic contributions are zero. The away side cross points are systematically higher than those at the near side, reflecting directly the amount of jet contribution at $\pi \pm \pi / 4$. The extremes of the distributions are not at $\pi / 2$ where the flow influence is maximal, instead they are shifted either to the left or the right due to the jet contribution. 


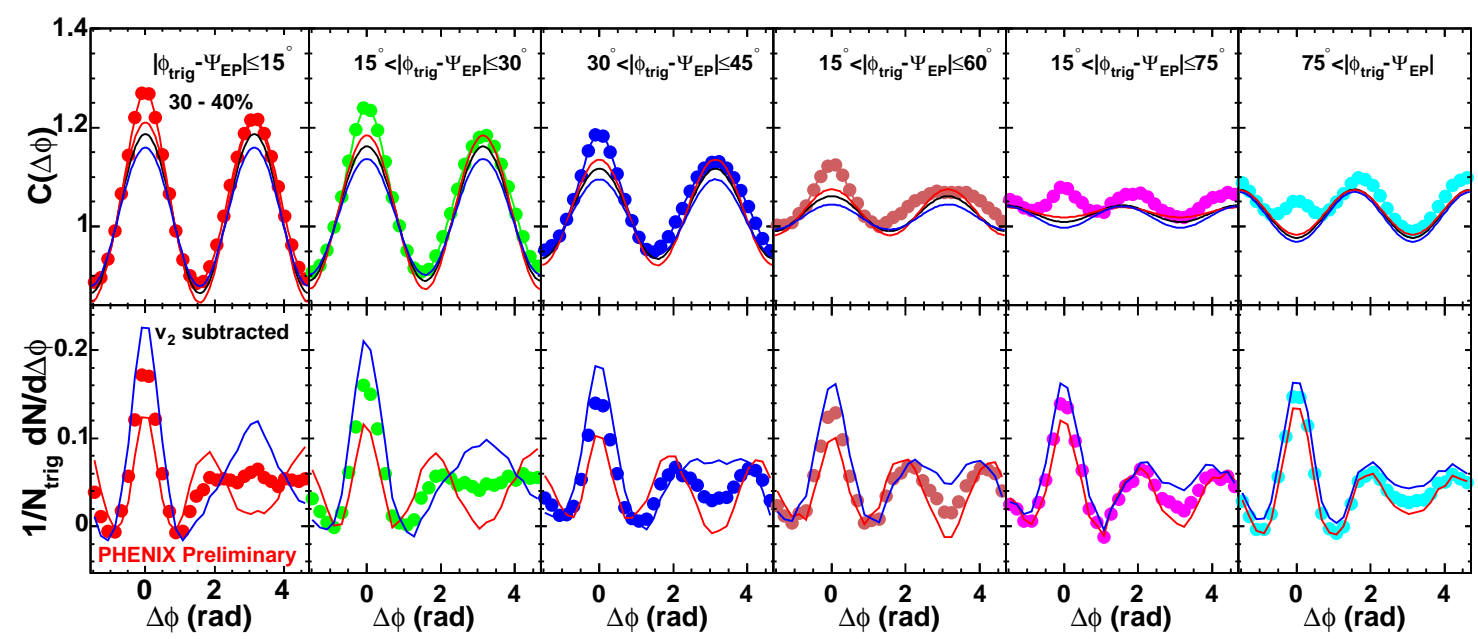

FIGURE 5. (top row) Correlation functions and (bottom row) background subtracted per-trigger yields for the 6 trigger direction bins.

The jet yields in each trigger direction (Fig.4b ) are obtained by subtracting the flow contribution. The only free parameter, $\xi$, is fixed by the ZYAM procedure from the integrated bin, and the flow terms in all six trigger bin are automatically fixed (Eq.2). Fig.5 shows the comparison of the the measured CFs and the calculated flow contributions for 30-40\% centrality bin. The systematic error bands correspond to the error of the RP $v_{2}$, propagated according to Eq.2. The size of the systematic errors is largest for in plane bin and smallest for the out of plane bin. RP dependence study helps to constrain the $v_{2}$ systematic when it is not dominated by the RP resolution ${ }^{1}$. In Fig. $4 \mathrm{~b}$, jet shapes for different bins show some subtle differences within the systematic error. We believe they are mostly due to the small $v_{4}$ terms which were not considered in current analysis.

In Fig.6, the difference of the per-trigger yield between in plane direction and out of plane direction: $\left(1 / N_{\text {trig }} \Delta N / \Delta \phi\right)_{\text {in }}-\left(1 / N_{\text {trig }} \Delta N / \Delta \phi\right)_{\text {out }}$ is plotted, and they are fitted with $c_{0}+c_{2} \cos 2 \Delta \phi+c_{4} \cos 4 \Delta \phi$ term. Indeed most of the variations can be accounted for by this function. There is a small excess on the away side relative the near side in $30-40 \%$ centrality bin. This excess could be the hint for the path length dependence of the away side jet modification.

\section{“REAPPEARANCE" OF THE AWAY SIDE JETS AT HIGH $P_{T}$}

The importance of high $p_{T}$ correlation is two fold. On the one hand, high $p_{T}$ jets are free from complicated intermediate $p_{T}$ physics (for example recombination), thus can serve as a cleaner probe of the medium. On the other hand, Studies of high $p_{T}$ jets can help to disentangle normal jet fragmentation from cherenkov gluons, shock wave or fragmentation of radiated gluons which become dominating at intermediate or low $p_{T}$.

\footnotetext{
${ }^{1}$ Since $v_{2}^{a}=v_{2, \text { raw }}^{a} /\langle\cos 2 \Psi\rangle$, the error of $v_{2}$ from RP resolution is independent of trigger direction.
} 


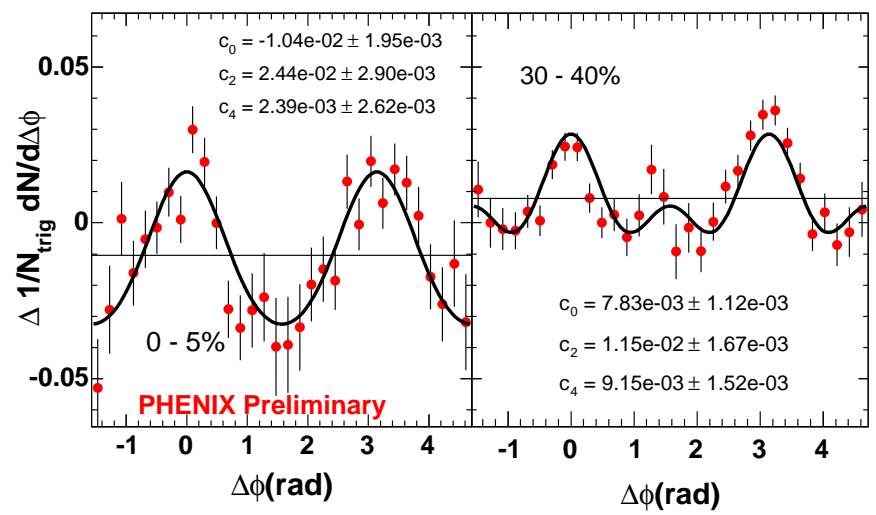

FIGURE 6. Difference of the in plane and out of plane per-trigger yield for a) $0-5 \%$ and b) $30-40 \%$ centrality bins.
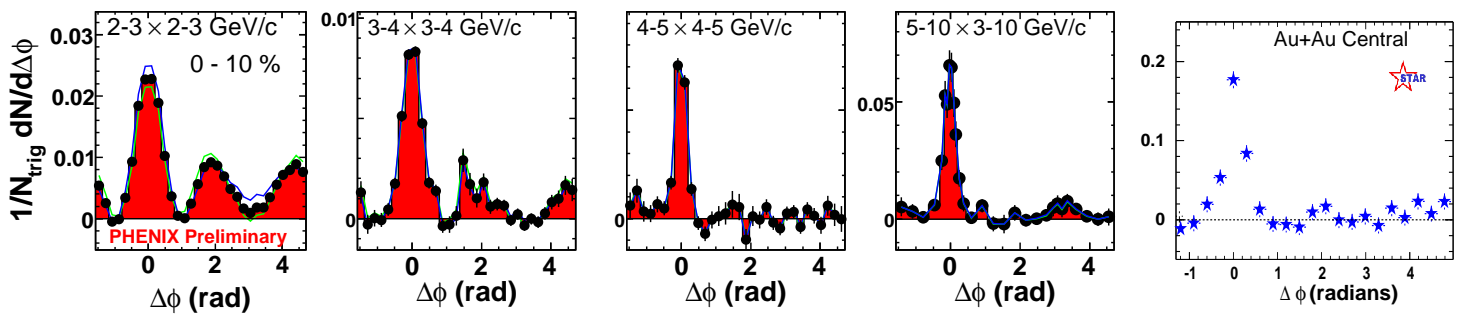

FIGURE 7. (left four panels) : per-trigger yield for different $p_{T}$ selection in $0-10 \%$ centrality bin. (right panel): per-trigger yield from STAR in $0-10 \%$ centrality bin [2].

Fig. 7 shows several CFs in successively higher $p_{T}$ ranges in $0-10 \%$ central $\mathrm{Au}+\mathrm{Au}$ collisions. The typical away side cone structure persists to $p_{T} \approx 4 \mathrm{GeV} / c$, but the edges of the cones become sharper and their magnitude drops. In $4-5 \times 4-5 \mathrm{GeV} / c$ bin, the relative flat away side shape does not rule out the cone shape, but it's magnitude must be significantly reduced.

As a comparison, in the most right panel of Fig. 7, we also show the hadron-hadron correlation from STAR [2]. The data are for 0-10\% most central $\mathrm{Au}+\mathrm{Au}$ with $4<$ $p_{T \text {,trig }}<6 \mathrm{GeV} / c$ and $2<p_{T \text {, assoc }}<p_{T \text {,trig }}$, and are comparable to the middle panel in Fig. 7. It is also almost comparable to the highest $p_{T}$ point in Fig. 3a, with the $p_{T}$ selection of the trigger and associated hadrons are swapped ${ }^{2}$. All three are qualitatively similar to each other. Interestingly, STAR's data are consistent with zero around $\pi$, but it seems to have a shoulder at $\pi \pm 1$ as suggested by the right panel of Fig. 7 .

In the highest $p_{T}$ bin of Fig.7, a peak structure seems to reemerge around $\pi$ on top of a flat background. To understand the physics behind the peak structure, we plot in Fig.8

\footnotetext{
${ }^{2}$ When the $p_{T}$ range of trigger and associated particle are swapped, the di-jet modification factor $I_{\mathrm{AA}}$

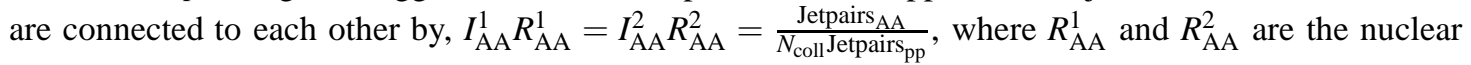
modification factor of the first and second particle, respectively. JetPairs $A$ A and JetPairs rp $_{\text {represent the }}$ average number of jet pairs in one A + A collision and one $\mathrm{p}+\mathrm{p}$ collision, respectively, respectively.
} 

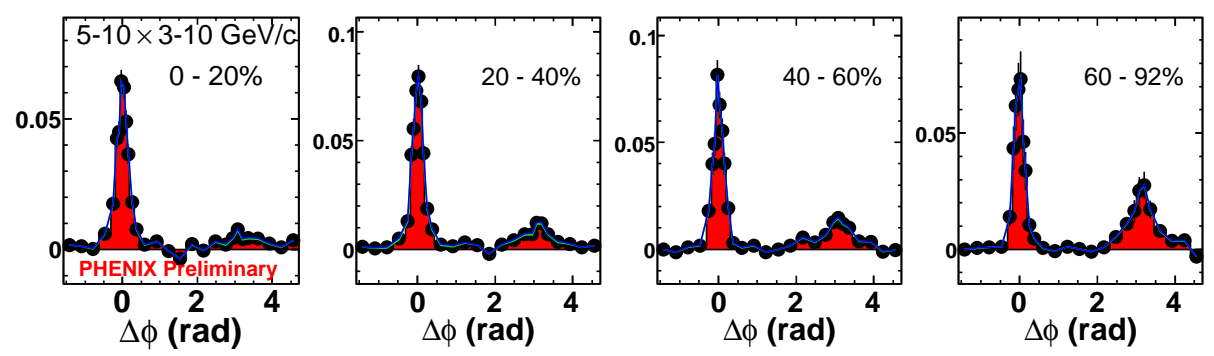

FIGURE 8. Centrality dependence of the per-trigger yield at high $p_{T}$
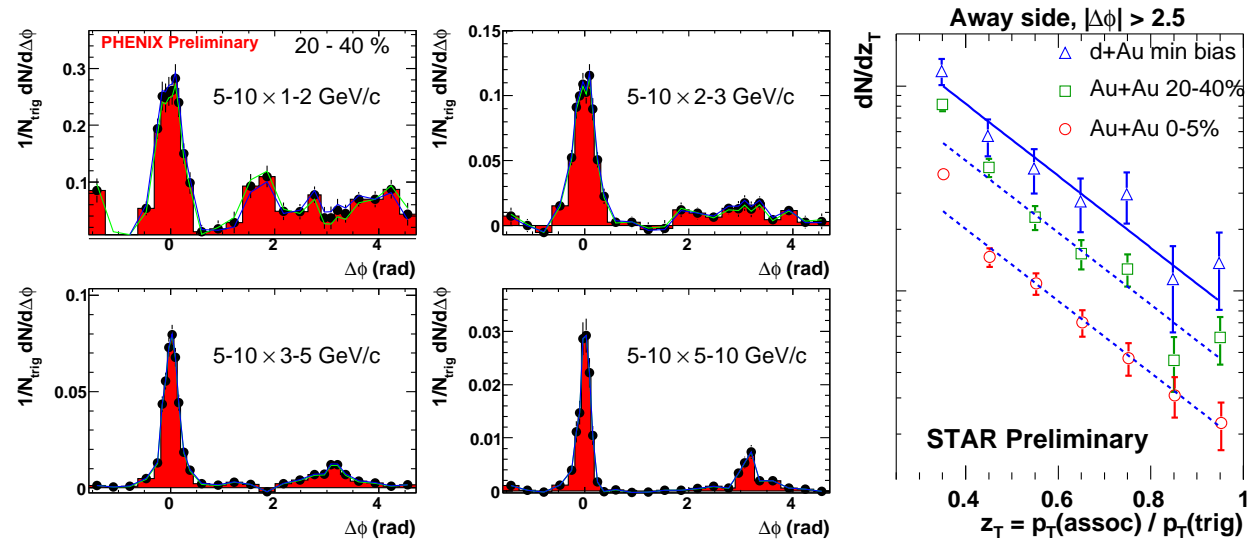

FIGURE 9. (left four panels): per-trigger yield for different $p_{T \text {,assoc }}$ in $20-40 \%$ centrality bin when trigger $p_{T}$ is fixed. (right panel) Away side jet fragmentation from STAR [17].

the centrality dependence of the $\mathrm{CF}$ for $5-10 \times 3-10 \mathrm{GeV} / \mathrm{c}$ selection. The away side peak exists in all centrality bins, although its magnitude is suppressed toward central collisions. At this point, it is hard to say whether the widths of the away peaks are also broadened in central collisions. On the other hand, there seems to be little change in both the shape and magnitude of the near side jet as function of centrality. These can be compared with recent di-jet results measured at much larger $p_{T}\left(8<p_{T \text {,trig }}<15\right.$ $\mathrm{GeV} / c$ and $6<p_{T \text {,assoc }} \mathrm{GeV} / c$ ) from STAR experiment [17], where the jet width and the shape of the fragmentation function are found to be independent of $p_{T}$ at fairly large $z$ $(z>0.4-0.5)$. In energy loss picture, the large $z$ requirement biases the detected away side jets to smaller energy loss, which biases detected jet towards surface, thus points to the picture where both jets are emitted tangential to the surface. If this scenario is true, we should recover the strong medium modification at low $z$ (by decreasing $p_{T, \text { assoc }}$ ). To check this, in Fig.9a we show the CF for various associated hadron $p_{T}$ with trigger $p_{T}$ fixed. The $\langle z\rangle$ of the associated hadron in the four panels are approximately $0.2,0.4$, 0.6 and 1. Clearly we see a stronger distortion of the away side jet shape at smaller $p_{T \text {,assoc }}$, the yields relative to the near side are also larger at smaller $p_{T \text {,assoc }}$. In fact, the fragmentation functions from STAR also suggest a significant deviation from the uniform scaling shape at $z \lesssim 0.4$ as shown in Fig.9b. It is important to measure the fragmentation function in full $z$ range in order to separate these two competing effects. 


\section{CONCLUSIONS}

Jet properties from hadron-hadron correlation have been studied as function of $p_{T}$, centrality and the angle relative to the reaction plane. Precise extraction of jet signal relies on experimental control on the flow backgrounds, which can be constrained by looking their reaction plane dependence. Jet shape and yield are found to be strongly modified at intermediate and low $p_{T}$. The interpretations of these modification, however, are complicated by various competing mechanisms. By increasing the $p_{T}$ for both triggering and associated hadrons, away side jet peak reappears but it's yield is suppressed. This might be due to the bias effect where the detected di-jets are emitted tangential to the surface.

\section{REFERENCES}

1. K. Adcox et al. [PHENIX Collaboration], Nucl. Phys. A 757 (2005) 184; J. Adams et al. [STAR Collaboration], Nucl. Phys. A 757 (2005) 102; B. B. Back et al. [PHOBOS Collaboration], Nucl. Phys. A 757 (2005) 28; I. Arsene et al. [BRAHMS Collaboration], Nucl. Phys. A 757 (2005) 1.

2. C. Adler et al. [STAR Collaboration], Phys. Rev. Lett. 90, 082302 (2003)

3. J. Adams et al. [STAR Collaboration], Phys. Rev. Lett. 95, 152301 (2005)

4. S. S. Adler et al. [PHENIX Collaboration], arXiv:nucl-ex/0507004.

5. J. Jia, J. Phys. G 31, S521 (2005)

6. N. N. Ajitanand et al., Phys. Rev. C 72, 011902 (2005)

7. Nathan Grau [PHENIX Collaboration], nucl-ex/0511046.

8. I. Vitev, arXiv:hep-ph/0506281.

9. I. M. Dremin, JETP Lett. 30 (1979) 140 [Pisma Zh. Eksp. Teor. Fiz. 30 (1979) 152].

10. V. Koch, A. Majumder and X. N. Wang, arXiv:nucl-th/0507063.

11. N. Armesto, C. A. Salgado and U. A. Wiedemann, arXiv:hep-ph/0411341.

12. J. Casalderrey-Solana, E. V. Shuryak and D. Teaney, arXiv:hep-ph/0411315.

13. J. Bielcikova, S. Esumi, K. Filimonov, S. Voloshin and J. P. Wurm, Phys. Rev. C 69, 021901 (2004)

14. A. Drees, H. Feng and J. Jia, Phys. Rev. C 71 (2005) 034909

15. S. S. Adler et al. [PHENIX Collaboration], Phys. Rev. Lett. 91 (2003) 172301

16. S. S. Adler et al. [PHENIX Collaboration], Phys. Rev. C 71 (2005) 051902

17. Dan magestro [STAR Collaboration], arXiv:nucl-ex/0510002. 